\title{
The Organisation of Floor in Meetings and the Relation with Speaker Addressee Patterns
}

\author{
Rieks op den Akker, Mariët Theune, Khiet Truong, Iwan de Kok \\ Human Media Interaction \\ University of Twente \\ Enschede, the Netherlands \\ \{infrieks|theune|k.p.truong|i.a.dekok\}@ewi.utwente.nl
}

\begin{abstract}
We present a procedure for conversational floor annotation and discuss floor types and floor switches in face-to-face meetings and the relation with addressing behavior. It seems that for understanding interactions in meetings an agent needs a layered floor model and that turn and floor changes are constrained by the activities and the roles that the agent and his conversational partners play in these activities. We present statistics about the addressee of the speaker and his role in the ongoing activity and a simple method that predicts the addressee using speaker role and floor state. The results support the expectation that information about the activity and the speaker's role will improve detection and interpretation of social signals from speaker addressee patterns in meetings.
\end{abstract}

\section{Categories and Subject Descriptors}

H.4 [Information Systems Applications]: Miscellaneous

\section{General Terms}

Theory

\section{Keywords}

conversational analysis, floor, addressing

\section{INTRODUCTION}

"The passing of the turn from one party to another is nearly the most obvious aspect of conversation" ([21], p.568). In "On getting a word in edgewise", Yngve suggests to adopt the concept of "the state of mind" in linguistics. "State of mind is postulated to contain all of the relevant contextual information, linguistic and non-linguistic, that the language user needs when carrying on communicative activity". The paper is often referred to as the source for what Yngve called the "back channel, over which the person who has the turn

Permission to make digital or hard copies of all or part of this work for personal or classroom use is granted without fee provided that copies are not made or distributed for profit or commercial advantage and that copies bear this notice and the full citation on the first page. To copy otherwise, to republish, to post on servers or to redistribute to lists, requires prior specific permission and/or a fee.

SSPW'10, October 29, 2010, Firenze, Italy.

Copyright 2010 ACM 978-1-4503-0174-9/10/10 ...\$10.00. receives short messages such as "yes" and "uh-huh" without relinquishing the turn" ([21], p.568). Thus, speaking is not the same as having the turn. Both the person who has the turn and the one who does not are engaged in both speaking and listening. Beside this important observation, Yngve makes a number of other remarks that are particularly interesting for modeling conversational behavior. Partners engaged in a conversation have their own idea about who has the turn. The "turn variable" (Yngve's phrasing) does not belong to "the conversation itself as a social phenomenon". When partners have different ideas about who has the turn it is difficult to find out who "really has the turn". Moreover, Yngve remarks, even if it was possible to find out who "really has the turn", it seems to be irrelevant for our purpose, to account for the partner's behavior, because this is motivated by the turn variable in the partner's (private) state of mind. A second remark Yngve makes is that "having the turn" is different from "having the floor". We will see examples of this from our corpus in section 2. Thus, there are several levels in the organisation of spoken interaction. A model of a conversational agent will have a "turn variable" and a "floor variable", holding information about who has the floor.

This paper is about the way turn and floor switches are organized in meetings. In formal meetings the floor organisation is related to the various meeting activities. Our interest is in building models useable for the mechanical analysis of multi-party conversations as well as in the construction of synthetic conversational agents that play a role in man machine interfaces or that play a character in virtual worlds, in games or in story telling systems.

The challenge of Social Signal Processing (SSP, see the work by Pentland [16] and for an overview [20] and [5]) is to build computational models and machines that identify social characteristics from easily observable signals of nonverbal human behavior. The technology could be useable for example for providing a discussion group with a "social mirror" (see Sturm et al. [18]).

Patterns in speaker turn sequences model the temporal order of the switches of the speaker role. They have been used as finger prints of the group, as cues for personal interactions and management styles. (See the work in small group research by Bales [1], Parker [15], and Gibson [6].) What makes speaker sequences attractive as a model for SSP is that they are so simple. The downside is that they abstract from a number of aspects of interaction that may be of interest for the task that we want the machine to perform. First, they assume one speaker at the time. Second, they treat all 
contributions as similar. In reality, contributions from different partners often come simultaneously, with very different flavors depending on the situation.

More involved models of conversations take into account who is being addressed by the speaker. Bales was one of the first who used measures such as talkativity and frequency of being addressed for dominance in small group interactions. Gibson measured personality traits by the frequency of the types of $\mathrm{P}$-shifts individual participants in a conversation are involved in. P-shifts are patterns consisting of two adjacent speaker addressee pairs [6]. The pattern AB$\mathrm{CA}$ signals a situation where a speaker $\mathrm{A}$ addresses $\mathrm{B}$ and where in the next turn another person (C) addresses A. Obviously, a model of turn taking that only consist of speakers, misses the information contained in P-shifts.

In this paper we discuss the organisation of activities in meetings and how they are related to speaker addressee patterns, and the roles of participants in these activities. We present a floor annotation procedure (similar to Chen et al.[3]) Our findings seem to imply a stack like model for the segmentation of floor reflecting the embedded structure of activities. We annotated who the speaker is addressing and we present statistics relating the floor state the meeting is in when a speaker contributes, his role and the type and role of the addressee. We present a simple rule based method for predicting the addressee of a speaker. This method uses the information about the floor state the meeting is in when the speaker starts speaking and about his role in the activity. This method improves other methods for addressee prediction that use statistical machine learning or a rule system.

\section{THE MEETING CORPUS}

The meeting data used for our analysis is drawn from a corpus of design project meetings (the AMI meeting corpus [2]). The meetings are role played by four people who act as partners in a design project. One of them has the role of project manager (PM). The PM takes care of the agenda and does the administration. We present some short fragments from AMI meeting ES2008a, the first meeting of a series of four meetings that a group has. We will return to these fragments when we discuss the annotations.

The four participants sit at a rectangular table, two at each side. The PM (Rose) welcomes everybody and goes through the agenda. She talks rather fast, glances now and then at her laptop and at the other three project members. The others are listening. After a short pause of 2 seconds, she changes topic.

(1) Fragment 1: lines 29-38

$\begin{array}{lll}29 & \text { Rose: Okay , } \\ 30 & \text { Rose: } & \text { we're gonna get to know each other a little } \\ & & \text { bit. } \\ 31 & \text { Rose: So um , } \\ 32 & \text { Rose: what we're gonna do is start off with um }\end{array}$
(1.0) (RESTART) let's start off with Amina

$\begin{array}{lll}33 & \text { Alima: } & \text { Alima . (CORRECTION) (Alima smiles) } \\ 34 & \text { Rose: } & \text { Um Alima, (Rose smiles at Alima) } \\ 35 & \text { Rose: } & \text { sorry } \\ 36 & \text { Alima: } & \text { [laugh] } \\ 37 & \text { Rose: } & (0.2) \text { Alima (.) } \\ 38 & \text { Rose: } & \text { um we're gonna do a little tool training, }\end{array}$

In the 1.0 second pause in line 32 of Fragment 1 Rose is looking around to select someone to start. When she looks at Alima, who sits in front of her across the table, she has made up her mind and she restarts, while bending over and pointing at Alima.

Alima's correction is an interruption without the intention to take over the floor. The PM responds by apologizing and correcting herself.

Fragment 2 shows another interruption.

(2) Fragment 2: lines 44-55

\begin{tabular}{|c|c|c|}
\hline 44 & Alima: & Okay \\
\hline & Alima: & $\begin{array}{l}\text { Um }(0.5) \text { I don't know which one of these I } \\
\text { have to bring with me. } \\
\text { (Alima stands up and brings the microphone } \\
\text { equipment with her. she turns to the white- } \\
\text { board.) }\end{array}$ \\
\hline & Ros & Probably both \\
\hline & & \\
\hline & $\begin{array}{l}\text { Alim } \\
\text { Alim }\end{array}$ & I'm supposed to draw my favourite a \\
\hline & Alima & But \\
\hline & Alima & \\
\hline & Alima: & Um I'm from the state of Maine in the US \\
\hline & Alima: & I'm doing nationalism studies, \\
\hline & Alim & \\
\hline & & artistic talents \\
\hline
\end{tabular}

Notice however that the short comment by Rose in line 46 "Probably both" is of quite a different type than the previous one. This contribution supports the action of the floor holder and is more ore less invited by Alima's remark, that has something of a request. And that's the way it is taken by Rose. Her action in a sense marks the request character.

While Alima is introducing herself, the PM makes a short note and that apparently raises a problem. She waits for the opportunity to ask the question and she found it here, after line 55, where Alima clearly finishes her sentence, turns over to the whiteboard, bound to start drawing her favorite animal.

\section{(3) Fragment 3: lines 56-58}

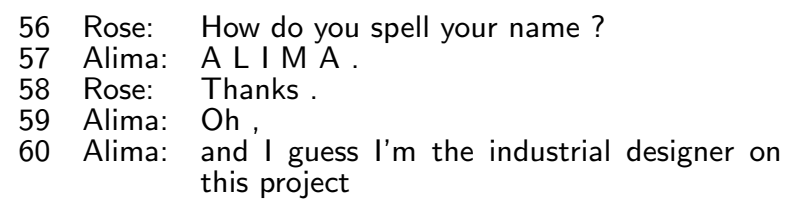

We are still in the middle of Alima's presentation and there is no discussion about who takes turn after the PM has closed the interchange with a thanks.

What we observe in these fragments is the organizing role of the project manager and the competence that participants share in having a meeting. It is the PM who is expected to start the meeting and who proposes to start with the partner's presentations. Although they follow a meeting scenario there is no script that prescribes them what to do. Rose attends Alima's presentation, sharing the attentional focus with the other listeners, but as a PM, she also has some administrative tasks to perform. When Alima has the floor the PM takes care to find an opportunity to interrupt Alima and to involve her in her administrative task. The PM's behavior signals her awareness of the fact that such an interruption is acceptable only at certain points and under particular circumstances and not without a certain form of politeness. What we also observe is that after this interruption that ends with the PM's polite thanking it is Alima 
who speaks next. She resumes her presentation. It is Alima who still holds the floor, although the interchange initiated by the PM is not on that floor, but on a different floor related to an activity that is controlled by the PM. This is what we call the management floor.

We will return to these fragments when we discuss the floor annotations. We now explain our notion of floor and the floor annotation procedure.

\section{ACTIVITY AND FLOOR}

The meaning of floor has shifted from turn to a more encompassing idea of attentive cognitive space. Yngve [21] already remarked that someone can take turn without taking the floor. Edelsky also claimed that floor and turn are not equivalent and defined floor as: "The acknowledged what'sgoing-on within a psychological time/space." ([4],p.405). Hayashi sees floor as "a kind of competence that is developed in the cognitive attentive space naturally or by mutual efforts when more than two persons interact with each other." ([8],p.2). Floor is related to topic, and floor changes reflect topic changes. Floor is related to the joint activities that people participate in. Jones and Thornborrow studied the way the conversational floor relates to the organization of talk in classroom activities. They stress that floor "represents a set of possibilities with identifiable activity-related constraints." (in [10],p.400). We adopt Hayashi's notions of floor, floor holder and floor supporter. The floor holder is the one who is the focus of attention of the ongoing topic. Floor supporters are attentive listeners that contribute to the activity on the floor. We emphasize the relation the floor has with the joint activities that participants in meetings are engaged in. Presentations, discussions, decision making, have their own typical organisation. Activities make other sub-activities necessary. Activities that plan other activities are special type of activities. Hayashi defines a whole hierarchy of different floor types. In our floor annotation we distinguish two types of floor: the single floor and the collaborative floor.

Four coders independently annotated AMI meeting IS1003b for floor type and floor holder, using Hayashi's description in [8]. Coders used the manual transcription of what has been said, as well as video and audio files. We discussed the findings and we produced a floor annotation manual to be used for further annotations.

\subsection{Floor annotation procedure}

There are two annotation layers: floor state and floor operations. There are two floor types. Single floor and Collaborative floor. A Single floor has a clear floor holder, the one who controls the floor, the one who is the main focus of attention, the one who is leading the activity. Others that participate in a single floor are not floor holders. Their contributions are floor support acts, backchannels, comments, short questions. A Collaborative floor is an episode where different people contribute but without someone who controls the floor. For all dialogue act segments we annotated the floor state as it is for the speaker at the start of the segment, using the following labels.

- $\mathbf{A}, \mathbf{B}, \mathbf{C}$ or $\mathbf{D}$ : If there is a single floor established, with a clear floor holder then use A,B,C or D indicating who is the floor holder.
Table 1: The types of floor operations based on [8] (FH is floor holder).

\begin{tabular}{|l|l|}
\hline code & operation \\
\hline YO & Unaddressed yield by FH \\
YT & Addressed yield by FH \\
HO & FH keeps floor \\
SW & FH switches topic \\
CL & Close floor by FH \\
\hline SU & Supports floor \\
FC & Successful Floor Claim \\
UC & Unsuccessful Floor Claim \\
\hline OT & Self-talk, non-floor speech \\
\hline
\end{tabular}

- O: Use O for Open or no floor. This is the floor state at the start of a meeting, or after an activity has finished, when the floor holder has given away the floor, without given it to someone else in particular. Usually there is a pause, silence, people are doing something for themselves (taking notes for example).

- $\mathbf{X}$ : Use X in all other cases. This label is also used for segments that are part of a collaborative floor.

The management floor is a special single floor type. Planning, summarizing, etc, are typical activities on this floor with the PM as floor holder. A typical single floor is a presentation floor, with the presenter as floor holder. A discussion is most of the time an activity with the flavor of a single floor, but sometimes it is more a collaborative floor.

With a contribution the actor can perform a floor operation, such as starting a new floor, or an attempt to change the established floor status. Table 1 summarizes the codes to be used for the floor operations.

Note that according to this floor model some floor operations can only occur in specific floor states, and some floor operations result in specific floor states.

\subsection{Agreements and difficulties in annotation}

Two coders applied the scheme to AMI meeting ES2008a. There are 382 dialogue act segments. Coders encountered a number of problems with the annotation task. Floor type agreement 343 of 382 segments, operation type agreement 297 of 382 segments. Discussion episodes in the meeting are sometimes hard to label by type of floor. Questions sometimes have a floor control function: the one who asks the question in a sense takes the floor but also gives the floor to the others. Sometimes they can be seen as just supportive. Annotators do not always agree about the floor holder then. A typical type of confusion we see in the two annotations of lines 55-59 (see Fragments 2 and 3 in section 2 ). The floor holder and floor operations that the two coders assigned to the fragment are as follows:

$$
\begin{aligned}
& B-H O ; B-F C ; A-S U ; A-C L ; B-H O \\
& B-H O ; B-S U ; B-H O ; B-S U ; B-H O
\end{aligned}
$$

According to both annotators at the start of lines 55 and $56 \mathrm{~B}$ (Alima) is the floor holder, and both agree that after 58 she is again (or still) the floor holder. The difference starts with the interpretation of Rose's question at line 56. Where the first annotator labels this as FC, a successful 
floor claim, after which Rose (A) becomes the floor holder, the second annotator labels Rose's question as an act that supports (SU) Alima's floor, and she remains floor holder in this view. We see a good example here of the different levels of analyses. The annotation scheme does not make a distinction between floor levels. It is clear that the PM does not take over the floor from Alima, who just started her presentation. This motivates the second annotation. On the other hand Rose's question is a real interruption, more than a short recipient response or a short comment or correction as Alima's in Fragment 1. The PM is asking a question to the floor holder that is related to the PM's concern. That motivates the 1st annotation: the PM becomes active floor holder and Alima's answer is a support act of this floor.

What is relevant here is how such pairs of turns (a question and an answer) or tripartite exchanges (a QA-pair followed by a thanks), or longer fragments are made to fit by negotiation between floor holder and interruptor in the ongoing conversation. What is clear from this example is that the activity that is interrupted was not finished and is resumed by the floor holder after the interruption. Similarly, we see that after a presentation or when a discussion is finished the PM resumes the management floor. It seems like a layered floor model that reflects the structural relations between activities and sub-activities as well as the relations between topics of discussions better fits the data than a strict sequential organisation. This has consequences for the turn-taking model.

\subsection{The multi-layered floor model}

Sacks et al. [17] describe how participants in a conversation construct turns at speaking and how they allocate them in a systematic way. Basis for their model (the SSJ model) is the notion of a turn constructional unit, the ending of which is marked as a transition relevant place, a possible place for recipients to take over the turn. According to the SSJ model the flow of conversations is governed by two mechanisms. First, the current speaker may select the next speaker, either by verbally or non-verbally addressing someone explicitly. Second, if the speaker did not select someone else then a listener may select him/herself and take the turn.

From our analysis it seems that in meetings roles and activities further constrain next speaker selection as well as the timing for possible turn switches. Activities like presentations and reports consist of pre-allocated longer turns. Partners (are expected to) share rules that regulate the turn taking in conversations embedded in these activities. The rituals of these activities determine what is considered acceptable and who is allowed to do what at what time. Presentations and reports are examples of "closed discourse units" in the sense of Houtkoop and Mazeland [9]. They describe how certain larger projects (stories, jokes, extended descriptions, pieces of advice) are produced in everyday informal conversations. Closed discourse units are activities larger than one turn-constructional unit and are projected as units from the beginning of their production. Our analysis supports their claim that turn construction not only operates through syntactical projection of a possible completion point, but that the type of activity is an important determinant to be taken into account.

Meetings have a layered floor structure with a stack like operation. If an activity is embedded in another activity and the embedded activity is closed, the floor holder of the embedding activity is the natural next speaker. On top of the management floor several activities are organized. Organizing activities on the management floor ("let's go around the table") have a clear impact on the way floor shifts are made and who takes part in these shifts. We see different regimes here: either the PM is given back the floor between presentations or the one who finished hands over to the next in line. Different types of leadership and personalities will be factors that cause these variations.

For successful participation and understanding of conversations embedded in meeting activities agents have to share knowledge about the structure and rituals that belong to these activities, and they have to know about the obligations and rights implied by the tasks that agents have in these activities.

\section{ADDRESSING IN MEETINGS}

It is the very nature of a communicative act that it is directed to others. Successful communication not only implies that a speaker's message is received and understood by a listener, but also that it was understood by the intended listener and that he has understood that he is the addressee. What communicative behavior is required to bring this about depends on the communicative situation. In particular, it depends on whether the speaker and listener already have a line of communication line open (see Lerner $([12])$. In this section we look at the relation between addressing and floor.

\subsection{Relation between floor state, role and ad- dressing mode}

Following Goffman the addressee is the participant "oriented to by the speaker in a manner to suggest that his words are particularly for them, and that some answer is therefore anticipated from them, more so than from the other ratified participants" ([7], p.9-10). Thus, the addressee is the listener the speaker has selected because he expects a response from that listener. The addressee often coincides with the one the speaker has selected to take the next turn. Most of the time in meetings speakers talk to the whole group. Sometimes however, a speaker addresses someone in particular, because what he has to say is particularly for him. For example when during a presentation the presenter says "next slide" this is clearly addressed to the one who is in control of the slides. In the meeting data that we analysed proper dialogue act segments, i.e. segments that express a speech act, are labeled with the addressee (see Jovanovic et al. [11]). Addressee is either Group or an individual, one of the participants (A, B, C, or D). For other utterance segments (labeled as Stalls, Fragments or Backchannels), the addressee is not annotated (label U).

For this meeting and one of the annotations (there is no systematic disagreement between the two floor type annotations) Tables 2 and 3 give the distribution of addressee types of dialogue acts in different situations when the speaker is floor holder or not. We have split off the cases where the speaker is the PM.

The last column in Table 2 contains the count of the special case where the PM is I-addressed. The last two columns in Table 3 contain the counts of the two special cases where the PM or the FH is I-addressed. From the figures in Tables 2 and 3 we can draw a number of interesting conclusions, 
Table 2: Distribution of types of addressing when the speaker is floor holder (this implies Single Floor state).

\begin{tabular}{|l|l|r|r|r|r|r|}
\hline \multicolumn{7}{|c|}{ Speaker $=$ Floor Holder } \\
\hline & & total & U & G & I & PM \\
\hline \multirow{2}{*}{ Single Floor } & SPKR=PM & 120 & 17 & 86 & 17 & - \\
& SPKR $\neq$ PM & 137 & 25 & 102 & 10 & 6 \\
\hline \hline & total & 257 & 42 & 188 & 27 & - \\
\hline
\end{tabular}

Table 3: Distribution of types of addressing when the speaker is not a floor holder (floor can be of various types).

\begin{tabular}{|l|l|r|r|r|r||r|r|}
\hline \multicolumn{9}{|c|}{ Speaker $\neq$ Floor Holder } \\
\hline & & total & U & G & I & PM & FH \\
\hline \multirow{2}{*}{ Open Floor } & SPKR $=$ PM & 5 & 2 & 1 & 2 & - & - \\
& SPKR $\neq$ PM & 12 & 6 & 5 & 1 & 1 & - \\
\hline \multirow{2}{*}{ Collab Floor } & SPKR $=$ PM & 1 & 1 & 0 & 0 & - & - \\
& SPKR $\neq$ PM & 9 & 3 & 2 & 4 & 0 & - \\
\hline \hline Single Floor $(\mathrm{PM})$ & SPKR $\neq \mathrm{PM}$ & 44 & 25 & 1 & 18 & 16 & 16 \\
\hline \multirow{2}{*}{ Single Floor $(\mathrm{OTH})$} & SPKR $=\mathrm{PM}$ & 26 & 12 & 4 & 10 & - & 10 \\
& SPKR $\neq \mathrm{PM}$ & 28 & 21 & 1 & 6 & 0 & 6 \\
\hline \hline Single Floor & total & 98 & 58 & 6 & 34 & - & 32 \\
\hline & total & 125 & 71 & 13 & 41 & - & - \\
\hline
\end{tabular}

with respect to the relation between floor holder and addressing, in the Single Floor state.

1. If speaker is floor holder: when speaker is the PM then contributions are much more I-addressed than when speaker is someone else. When speaker is not the PM then an I-addressed contribution is directed to the PM.

2. If speaker is not floor holder: $1 / 3$ of the contributions is I-addressed, and $2 / 3$ are improper DAs (labeled U). There are almost no G-addressed acts, and the I-addressed acts are almost always addressed to the floor holder.

Knowing who is the floor holder and who is speaking thus gives substantial support in finding who is being addressed by the speaker. Based on the statistics in Tables 2 and 3 we can compute the accuracy (percentage correct) of a simple rule based method for addressee prediction, assuming knowledge of floor state and floor holder. Figure 1 shows the algorithm. The accuracy is $85 \%$. Other methods for addressee prediction have been tested: a RuleBased method, a GazeBased method that only uses speaker's gaze, and a method that combines the rule based method with speaker's gaze information (see [14]). If we apply these methods to the same data we obtain accuracies of: $81 \%$ (rule), $83 \%$ (gaze), and $84.6 \%$ (combined). Thus for addressee prediction knowledge about floor state and floor holder compensates the lack of knowledge of dialogue act type, previous speaker, and speaker's gaze.

We tested this method on another meeting (AMI IS1003b). The number of DAs is 693, with 447 proper DAs; of which 177 are G-addressed and 270 are I-addressed. It was annotated for floor type by three annotators, all agreed on $417 / 693$. For this meeting, the accuracy of the method is $58 \%$. The combined rule-based method achieved an accuracy of $52 \%$ on this meeting. Thus also for this meeting
Figure 1: Algorithm for addressee prediction based on known floor type and floor holder. Between brackets the number of correct answers compared to the number of cases (the number of proper dialogue act segments).

If $($ Speaker $=$ FloorHolder $)$

return Group; (188/215)

Else \{

If (OpenFloor or CollabFloor)

return Group; (8/15)

If (SingleFloor) \{

If (FloorHolder $=\mathrm{PM}$ )

return PM; (16/19)

If (FloorHolder !=PM)

\}

\} 
knowledge of floor state and role of speaker helps predicting who the speaker is addressing.

Based on these findings we expect that automatic detection of floor state and knowledge about the speaker's role will improve addressee detection and help in understanding social interactions in meetings. Evidence that the addressee's role and topic information improve the performance of statistical addressee prediction methods was also reported in [13].

\section{CONCLUSIONS}

Our investigations of a corpus of face-to-face meetings has confirmed that floor is a useable construct in a model for a number of social phenomena in meetings, such as turntaking and addressing. Politeness rules as well as rights and obligations that follow from tasks that people have in the a collaborative activity play a role in assessment of interruptions and other floor operations in meetings. Building technology for understanding social cues in multi-party conversations as well as for building social agents requires a model of the agent's state of mind that contains variables for turn, as well as for the floor state.

Natural language dialogue systems often already have a notion of turn built in and synthetic characters that show "natural listening behavior" are underway. Occasionally, systems for multi-party dialogues with embodied agents contain a model of attention, and of floor and floor operations (see Traum and Rickel [19]). Further analysis of natural conversational human behavior describing what parameters of the situational context and the interpersonal relations between partners are relevant in deciding the meaning of social signals is needed.

\section{ACKNOWLEDGMENTS}

This work has been funded in part by the European Community's 7th Framework Programme [FP7/2007- 2013] under the grant agreement no 231287 (SSPNet).

\section{REFERENCES}

[1] R. Bales. Interaction process analysis: A method for the study of small groups. Addison-Wesley Press, Cambridge, 1950.

[2] J. C. Carletta. Unleashing the killer corpus: experiences in creating the multi-everything AMI meeting corpus. Language Resources and Evaluation, 41(2):181-190, May 2007.

[3] L. Chen, M. Harper, A. Franklin, T. R. Rose, I. Kimbara, Z. Huang, and F. Quek. A multimodal analysis of floor control in meetings. In S. Renals, S. Bengio, and J. e. Fiscus, editors, Machine Learning for Multimodal Interaction. Springer Berlin/Heidelberg, Book Series Lecture Notes in Computer Science, Volume 4299, 2006.

[4] C. Edelsky. Who's got the floor? Language and Society, 10(3):383-421, 1981.

[5] D. Gatica-Perez. Automatic nonverbal analysis of social interaction in small groups: A review. Image Vision Comput., 27(12):1775-1787, 2009.

[6] D. R. Gibson. Participation shifts: Order and differentiation in group conversation. Social forces, 81(4):1335-1381, 2003
[7] E. Goffman. Replies and responses. In Forms of Talk, pages 5-77. Philadelphia: University of Pennsylvania Press, 1981.

[8] R. Hayashi. Floor structure of English and Japanese conversation. Journal of Pragmatics, 16:1-30, 1991.

[9] H. Houtkoop-Steenstra and H. Mazeland. Turns and discourse units in everyday conversation. Journal of Pragmatics, 9:595-619, 1985.

[10] R. Jones and J. Thornborrow. Floor, talk and the organization of classroom activities. Language in Society, 33:399-423, 2004.

[11] N. Jovanovic, R. op den Akker, and A. Nijholt. Addressee identification in face-to-face meetings. In Proceedings of 11th Conference of the European Chapter of the Association for Computational Linguistics (EACL), Trento, Italy, 2006.

[12] G. H. Lerner. Selecting next speaker: The context-sensitive operation of a context-free organization. Language in Society, 32:177-201, 2003.

[13] H. op den Akker and R. op den Akker. Are you being addressed? - real-time addressee detection to support remote participants in hybrid meetings. In (Steve Young and Donna Byron eds.) Proceedings of SIGDIAL 2009, 2009.

[14] R. op den Akker and D. Traum. A comparison of addressee detection methods for multiparty conversations. In Proceedings of DiaHolmia, 13th Workshop on the Semantics and Pragmatics of Dialogue, 2009.

[15] K. C. H. Parker. Speaking turns in small group interaction: A context-sensitive event sequence model. Journal of Personality and Social Psychology, 4(6):965-971, 1988.

[16] A. Pentland. Honest Signals. The MIT Press, 2008.

[17] H. Sacks, E. Schegloff, and G. Jefferson. A simplest systematics for the organization of turn-taking for conversation. Language, 50:696-735, 1974.

[18] J. Sturm, O. H.-v. Herwijnen, A. Eyck, and J. Terken. Influencing social dynamics in meetings through a peripheral display. In ICMI '07: Proceedings of the 9th international conference on Multimodal interfaces, pages 263-270, New York, NY, USA, 2007. ACM.

[19] D. Traum and J. Rickel. Embodied agents for multiparty dialogue in immersive virtual worlds. In Proceedings of the first international joint conference on Autonomous agents and multiagent systems: part 2. ACM Press, 2002.

[20] A. Vinciarelli, M. Pantic, and H. Bourlard. Social signal processing: Survey of an emerging domain. Image and Vision Computing Journal, 27, no. 12, December 2009.

[21] V. Yngve. On getting a word in edgewise. In Papers from the sixth regional meeting of the Chicago Linguistic Society, pages 567-77, Chicago: Chicago Linguistic Society, 1970. 